\title{
Formulation and delivery of itraconazole to the brain using a nanolipid carrier system
}

This article was published in the following Dove Press journal:

International Journal of Nanomedicine

2 May 2014

Number of times this article has been viewed

Wei Meng Lim'

Paruvathanahalli

Siddalingam Rajinikanth ${ }^{2}$

Chitneni Mallikarjun'

Yew Beng Kang'

'School of Pharmacy, International Medical University, Kuala Lumpur, Malaysia; ${ }^{2}$ School of Pharmacy, Taylor's University, Selangor, Malaysia
Correspondence: Wei Meng Lim School of Pharmacy, International Medical University, 126, Jalan Jalil Perkasa 19, Bukit Jalil, 57000, Kuala Lumpur, Malaysia Tel +60327317486 Fax +60 386567229

Email weimeng_lim@imu.edu.my
Abstract: The objectives of this study were to develop and characterize itraconazole (ITZ)loaded nanostructured lipid carriers (NLCs) and to study their potential for drug delivery into the brain. Precirol ${ }^{\circledR}$ ATO 5 and Transcutol ${ }^{\circledR}$ HP were selected as the lipid phase, and Tween ${ }^{\circledR}$ 80 and Solutol ${ }^{\circledR}$ HS15 as surfactants. The ITZ-NLCs were prepared by a hot and high-pressure homogenization method. The entrapment efficiency for the best formulation batch was analyzed using high-performance liquid chromatography and was found to be $70.5 \% \pm 0.6 \%$. The average size, zeta potential, and polydispersity index for the ITZ-NLCs used for animal studies were found to be $313.7 \pm 15.3 \mathrm{~nm},-18.7 \pm 0.30 \mathrm{mV}$, and $0.562 \pm 0.070$, respectively. Transmission electron microscopy confirmed that ITZ-NLCs were spherical in shape, with a size of less than 200 $\mathrm{nm}$. Differential scanning calorimetry and X-ray diffractometry analysis showed that ITZ was encapsulated in the lipid matrix and present in the amorphous form. The in vitro release study showed that ITZ-NLCs achieved a sustained release, with cumulative release of $80.6 \% \pm 5.3 \%$ up to 24 hours. An in vivo study showed that ITZ-NLCs could increase the ITZ concentration in the brain by almost twofold. These results suggest that ITZ-NLCs can be exploited as nanocarriers to achieve sustained release and brain-targeted delivery.

Keywords: lipid nanoparticles, brain delivery, nanostructured lipid carrier

\section{Introduction}

Solid lipid nanoparticles (SLNs) attract much attention as a potential drug carrier because they offer many advantages compared to other carrier systems such as polymeric nanoparticles, nanoemulsions, and liposomes. The material for lipid nanoparticles is a solid lipid that remains in the solid state at room temperature. ${ }^{1}$ SLNs provide a better safety profile, as the materials used are biodegradable and biocompatible. ${ }^{2}$ The drug molecules that are incorporated into the lipid matrix are slowly released over time to achieve controlled-release. ${ }^{3,4}$ The solid lipid core increases drug stability by protecting the drug from degradation. ${ }^{1,4}$ Furthermore, formulation of lipid nanoparticles offers feasibility for large-scale production using methods such as highpressure homogenization. ${ }^{4}$

The so-called nanostructured lipid carrier (NLC) is a second-generation SLN. While SLN is prepared using a solid lipid alone, NLCs are prepared by mixing solid lipid with liquid lipid. Using solid lipid alone tends to form a perfect crystal lattice, which will limit the drug-loading capacity. Initially, the crystal structure of SLNs is disordered - for example, they exist in the $\alpha$ - and $\beta$ '-forms; however, during storage, the crystal structure will transform into its low-energy and more ordered $\beta$-form. This results in the expulsion of drug molecules from the crystal lattice. ${ }^{5}$ This problem is 
solved by mixing solid and liquid lipid together to generate an imperfect crystal structure with more space to accommodate drug molecules. ${ }^{6}$ Therefore, NLCs overcome the problems of instability and low loading capacity of SLNs.

Itraconazole (ITZ) is an antifungal drug that is widely used to treat various fungal infections, including those of Aspergillus, Cryptococcus, Candida, Blastomyces, and Histoplasma. ${ }^{7,8}$ It acts by disrupting the synthesis of ergosterol to prevent the formation of the fungal cell membrane. ${ }^{9}$ ITZ is classified in the biopharmaceutical classification system as a class II drug due to its poor water solubility. It has an n-octanol/water partition coefficient of 5.66 at $\mathrm{pH} 8.1 .^{10}$ It is reported to have solubility of $1 \mathrm{ng} / \mathrm{mL}$ at $\mathrm{pH} 7$ and of $4 \mu \mathrm{g} / \mathrm{mL}$ at $\mathrm{pH} 1 .{ }^{11}$ To overcome this problem, the current marketed intravenous formulation involves the complexation of ITZ with 2-hydroxypropyl- $\beta$-cyclodextrin (H $\beta C D)$. For every $10 \mathrm{mg}$ of ITZ, as much as $400 \mathrm{mg}$ of $\mathrm{H} \beta C \mathrm{CD}$ complex is required for complete solubilization. ${ }^{7}$ The product is of limited use for patients with severe renal impairment due to the low clearance of $\mathrm{H} \beta \mathrm{CD}$, as this will increase the risk of $\mathrm{H} \beta C D$ toxicity, leading to splenomegaly and ataxia. ${ }^{12}$ There is also a report that suggests that $\mathrm{H} \beta C D$ causes pancreatic adenocarcinoma in rats. ${ }^{13}$

Drug delivery across the blood-brain barrier (BBB) is very important, because the incidence for central nervous system-related disease, such as Parkinson's disease, brain cancer, stroke, Alzheimer's disease, and epilepsy, is increasing. The current treatment for central nervous system fungal infection, such as with Cryptococcus neoformans, Aspergillus spp., and Candida spp., is poor due to the presence of the BBB, which limits the access of antifungal drug molecules to the brain. ${ }^{14}$ Due to the nature of this unique barrier, approximately $98 \%$ of neurotherapeutic molecules are unable to cross the BBB. ${ }^{15}$

The endothelial cells of brain capillaries have higher amounts of mitochondrial content than those of peripheral capillaries; present without fenestrae; have low pinocytotic activity; and show the formation of tight junctions. ${ }^{16}$ The tight junctions known as zonula occludens are formed by the interaction of transmembrane proteins such as occludin and claudins adjacent to endothelial cells. This structure restricts the movement of substances through the paracellular pathway. ${ }^{17,18}$ Moreover, the endothelial cells in the brain capillaries express high levels of efflux pumps, such as P-glycoprotein (P-gp), multidrug resistance protein-1, and breast cancer resistance protein, which actively eliminate drugs out of the central nervous system. ${ }^{19}$

Several properties of ITZ have contributed to its poor permeability across the BBB. Firstly, ITZ has a large molecular weight of $705 \mathrm{Da}$. The upper limit of molecular weight for efficient permeability through the $\mathrm{BBB}$ is about 300-400 Da. ${ }^{20}$ Secondly, ITZ exhibits a high degree of binding to serum albumin. Binding to the large serum protein could impede the permeability across the BBB. ${ }^{14}$ Thirdly, ITZ is also a substrate of the P-gp pump along the BBB. ${ }^{21}$ This P-gp efflux pump will pump out the ITZ from the BBB. Kinetic studies for ITZ in both animal models and humans have shown that ITZ at a concentration of less than $1 \%$ is nearly undetectable in cerebrospinal fluid. ${ }^{14}$ All of the above factors contribute to the poor permeability of ITZ across the BBB.

In previous studies, research groups introduced ITZloaded nanoparticles for different purposes. Chen et al formulated ITZ nanoparticles using human serum albumin for parenteral delivery ${ }^{7}$ Kim et al designed ITZ-loaded lipid nanoparticles to achieve controlled release when administered intravenously; ${ }^{13}$ Pardeike et al formulated ITZ-loaded lipid nanoparticles for pulmonary application; ${ }^{10}$ and Duret et al embedded ITZ-loaded nanoparticles in inhalable microparticles for the treatment of pulmonary aspergillosis. ${ }^{22}$ However, to the best of our knowledge, the preparation of ITZ-loaded lipid nanoparticles for brain-targeted delivery has not yet been evaluated.

The application of lipid nanoparticles to brain delivery has been tested by various research groups. Yang et al formulated lipid nanoparticles containing the anticancer drug camptothecin and studied their effect on brain-targeted delivery. The results showed that camptothecin lipid nanoparticles had a better distribution to the organs and longer retention time and exhibited a higher concentration in the brain compared to camptothecin drug solution. ${ }^{23}$ In another study, Zara et al studied the biodistribution of SLNs loaded with doxorubicin. Pharmacokinetic studies in rats showed that the SLN formulation achieved higher drug concentrations in the lung, spleen, and brain compared to the marketed doxorubicin solution. ${ }^{24}$

\section{Materials and methods Materials}

ITZ was a gift from RA Chem Pharma Ltd (Hyderabad, India). Precirol ${ }^{\circledR}$ ATO 5 (glyceryl distearate) and Transcutol ${ }^{\circledR}$ HP ([TRA] diethylene glycol monoethyl ether) were a gift from Gattefossé (Lyon, France). Tween ${ }^{\circledR} 80$ (TW80) was purchased from Merck KGaA (Darmstadt, Germany), and Solutol $^{\circledR}$ HS15 ([HS15] polyoxyl-15-hydroxystearate) was purchased from BASF (Ludwigshafen, Germany). Fetal bovine serum was purchased from i-DNA Biotechnology Pte Ltd (Singapore). High-performance liquid chromatography 
(HPLC)-grade acetonitrile was purchased from Thermo Fisher Scientific (Waltham, MA, USA).

\section{Preparation of ITZ-loaded NLCs}

ITZ-loaded NLCs (ITZ-NLCs) were prepared using a highpressure homogenization (HPH) method. The lipid phase, consisting of Precirol ATO 5, TRA, and ITZ, was mixed and heated to $80^{\circ} \mathrm{C}$ until the lipid had completely melted. To prepare the aqueous phase, the desired amount of surfactants were added into $50 \mathrm{~mL}$ deionized water, heated to $80^{\circ} \mathrm{C}$, and continuously stirred until all the surfactants had completely dissolved. Subsequently, the hot lipid phase was poured into the aqueous phase and homogenized at a speed of 11,000 rpm (Ultra-Turrax Basic T25; IKA, Wilmington, NC, USA) for 2 minutes to form an emulsion. The emulsion was quickly passed through HPH (EmulsiFlex-C3; Avestin, Inc., Ottawa, ON, Canada) with the pressure at 1,500 bar for five cycles. The final product was cooled to room temperature to form ITZ-NLCs. The product was then placed in an Amicon ${ }^{\circledR}$ Ultra-4 filtration unit with a molecular weight cut-off of 30,000 Da (EMD Millipore, Billerica, MA, USA) and centrifuged for 10 minutes at 3,000 $\times g$ (Centrifuge 5702; Eppendorf, Hamburg, Germany). Lipid nanoparticles free of unloaded drug were obtained. The compositions of the various formulations prepared for use in the study are shown in Table 1.

\section{Determination of entrapment efficiency}

The entrapment efficiency (EE) was measured based on free drug concentration in the formulation by using

Table I Compositions for the preparation of itraconazole-loaded nanostructured lipid carriers

\begin{tabular}{|c|c|c|c|c|c|c|}
\hline \multirow[t]{2}{*}{ Formulation } & \multirow[t]{2}{*}{$\begin{array}{l}\text { Drug } \\
(\% \mathrm{w} / \mathrm{w})\end{array}$} & \multicolumn{2}{|c|}{$\begin{array}{l}\text { Lipid } \\
(\% \text { w/w) }\end{array}$} & \multicolumn{2}{|c|}{$\begin{array}{l}\text { Surfactant } \\
(\% \mathrm{w} / \mathrm{w})\end{array}$} & \multirow[t]{2}{*}{$\begin{array}{l}\text { Water } \\
(\% \mathrm{w} / \mathrm{w})\end{array}$} \\
\hline & & PRE $^{\mathbf{a}}$ & TRA $^{a}$ & TW80 & HSI5c & \\
\hline $\mathrm{FI}$ & 0.06 & 1 & 1 & 2 & - & 95.94 \\
\hline F2 & 0.06 & I & I & - & 2 & 95.94 \\
\hline F3 & 0.06 & 1 & 1 & I & I & 95.94 \\
\hline F4 & 0.06 & I & I & 4 & - & 93.94 \\
\hline F5 & 0.06 & 1 & 1 & - & 4 & 93.94 \\
\hline F6 & 0.06 & 1 & I & 2 & 2 & 93.94 \\
\hline F7 & 0.06 & 1.5 & 0.5 & 2 & - & 95.94 \\
\hline F8 & 0.06 & 1.5 & 0.5 & - & 2 & 95.94 \\
\hline F9 & 0.06 & 1.5 & 0.5 & 1 & I & 95.94 \\
\hline FIO & 0.06 & 1.5 & 0.5 & 4 & - & 93.94 \\
\hline FII & 0.06 & 1.5 & 0.5 & - & 4 & 93.94 \\
\hline $\mathrm{FI} 2$ & 0.06 & 1.5 & 0.5 & 2 & 2 & 93.94 \\
\hline
\end{tabular}

Notes: a'Gattefossé (Lyon, France); 'Merck KGaA (Darmstadt, Germany); 'BASF (Ludwigshafen, Germany).

Abbreviations: PRE, Precirol ${ }^{\circledast}$ ATO 5; TRA, Transcutol ${ }^{\circledast}$ HP; TW80, Tween ${ }^{\circledast} 80$; $\mathrm{HSI}$, Solutol ${ }^{\otimes} \mathrm{HSI} 5$. the ultra-filtration method. ${ }^{10}$ The amount entrapped was determined by subtracting the free drug concentration from the total drug added. The sample was placed in the Amicon Ultra-4 filtration unit with molecular weight cut-off of 30,000 Da and centrifuged for 10 minutes at $3,000 \times g$. The filtrate was analyzed by HPLC method (1200 HPLC series; Agilent Technologies, Santa Clara, CA, USA) with a C-18 analytical column $(5 \mu \mathrm{m}, 4.6 \times 150 \mathrm{~mm}$; Phenomenex, Torrance, CA, USA) The mobile phase comprised acetonitrile and deionized water (70:30, v/v\%); the flow rate was $1 \mathrm{~mL} /$ minute; and the detection wavelength was $262 \mathrm{~nm}$. The EE was calculated based on the equation below:

$$
\text { EE }(\%)=\frac{\text { Weight of ITZ added }- \text { Free ITZ }}{\text { Weight of ITZ added }} \times 100 \% \text {. (1) }
$$

\section{Particle size and zeta potential analysis}

The average particle size, ZP, and polydispersity index of ITZ-NLCs was measured by photon correlation spectroscopy (Zetasizer Nano ZS; Malvern Instruments, Malvern, UK).

\section{Transmission electron microscopy}

The shape of the ITZ-NLCs was examined using a transmission electron microscope (7100S; Hitachi Ltd, Tokyo, Japan). The sample was diluted with deionized water and placed on a carbon grid. Staining was performed using $2 \%$ uranyl acetate.

\section{Differential scanning calorimetry}

Differential scanning calorimetry (DSC) was performed to study the degree of crystallinity of the lipid. The DSC measurement was performed using a Mettler Toledo DSC823e (Mettler-Toledo International, Inc., Greifensee, Switzerland). ITZ-NLCs were lyophilized to obtain dry powder for DSC analysis. The sample was weighed and sealed in a $40 \mu \mathrm{L}$ aluminum crucible. The heating range was set at $30^{\circ} \mathrm{C}-200^{\circ} \mathrm{C}$ at the rate of $10^{\circ} \mathrm{C}$ per minute, and then cooled back to $30^{\circ} \mathrm{C}$ under nitrogen flow.

\section{X-ray diffraction study}

The X-ray diffraction (XRD) patterns for ITZ, ITZ-NLCs, and blank NLCs were analyzed using an X-ray diffractometer (X'Pert PRO PW 3040 MRD; PANalytical, Almelo, the Netherlands). Scanning for the freeze dried sample was conducted over $2 \theta$, ranging from $5^{\circ}$ to $40^{\circ}$, at a voltage of $45 \mathrm{kV}$ and a current of $40 \mathrm{~mA}$. 


\section{In vitro release study}

The in vitro release was tested using a dialysis membrane with a molecular weight cut-off of $12,000 \mathrm{Da}$, using the method reported by Chen et al. ${ }^{7}$ An ITZ-NLC sample containing $1 \mathrm{mg}$ of encapsulated ITZ was loaded into a dialysis bag. The bag was immersed into $50 \mathrm{~mL}$ phosphate-buffered saline ( $\mathrm{pH} 7.4$ ) containing $10 \% \mathrm{v} / \mathrm{v}$ fetal bovine serum (physiological medium). The temperature of the shake incubator was maintained at $37^{\circ} \mathrm{C}$ and the rotation speed was set at $100 \mathrm{rpm}$. The sample was withdrawn at time intervals of 1 , $2,4,6,8,10,12,16,20$, and 24 hours, and the same amount of receptor medium was replaced to the receptor chamber to maintain a constant volume. Similar studies were conducted on ITZ solution to test the permeability of the drug through the dialysis bag. All samples were analyzed using HPLC.

\section{In vivo brain ITZ concentration study}

The animal study was performed in accordance with the Animal Ethical Guidelines for Investigations in Laboratory Animals and was approved by the Ethical Committee for Animal Experimentation of the International Medical University, Kuala Lumpur, Malaysia. The in vivo brain distribution study was performed on mice, each being approximately $20 \mathrm{~g}$ in weight. The mice were divided into two groups. The first group was treated with ITZ complex with $\mathrm{H} \beta C D$, while the second group received ITZ-NLCs. The dose of ITZ administered to both groups was $20 \mathrm{mg} / \mathrm{kg}$ body weight. ITZ-NLCs from formulation batch F12 were used in this study. Specifically, $20 \mathrm{mg}$ of ITZ-NLC formulation F12 containing $0.4 \mathrm{mg}$ of ITZ was suspended in $0.2 \mathrm{~mL}$ of sterile water for injection, and then administered intravenously through the tail vein. The animals were sacrificed after 2, 4, 8, or 12 hours, and the brain was harvested and homogenized. To a $200 \mu \mathrm{L}$ homogenized sample, $200 \mu \mathrm{L}$ of acetonitrile was added to precipitate the protein and the sample was centrifuged at 5,000 rpm for 10 minutes before collection of the supernatant.

The supernatant was then analyzed using the HPLC method reported by Redmann and Charles for plasma estimation of ITZ with modifications. ${ }^{25}$ The HPLC system (Agilent 1260 HPLC series) used was equipped with a sample injection port fitted with a $100 \mu \mathrm{L}$ sample loop and a fluorescence detector. The analytical column used was the Phenomenex C-18 analytical column $(5 \mu \mathrm{m}, 4.6 \times 150 \mathrm{~mm})$. The mobile phase was made up of acetonitrile and deionized water $(70: 30, \mathrm{v} / \mathrm{v} \%)$ at the flow rate of $1 \mathrm{~mL} /$ minute. The injection volume was $100 \mu \mathrm{L}$, and the fluorescent detector was set at excitation wavelength $250 \mathrm{~nm}$ and emission wavelength $380 \mathrm{~nm}$.
The calibration curves were constructed by spiking drug-free brain homogenates with known amounts of ITZ at concentrations of $0.1-10 \mu \mathrm{g} / 0.2 \mathrm{~g}$. The standard calibration curve $(n=6)$ was found to be linear over the concentration range used with a correlation coefficient of 0.999 . The samples were quantified using the peak area. The limit of quantification was set at $100 \mu \mathrm{g} / 0.2 \mathrm{~g}$ of brain homogenate, which was the lowest concentration used in constructing the standard calibration curve, having an accuracy and precision value below $\pm 20 \%$. The limit of detection was $30 \mathrm{ng} / 0.2 \mathrm{~g}$ of brain homogenate. To a $200 \mu \mathrm{L}$ homogenized sample, $200 \mu \mathrm{L}$ of acetonitrile was added to precipitate the protein and the sample was centrifuged at 5,000 rpm for 10 minutes to collect the supernatant, which was analyzed using the HPLC system. The results were compared with that of aqueous ITZ standard with similar concentration and the recovery percentage was calculated. The average recovery was calculated to be $92.14 \% \pm 3.21 \%$.

\section{Results and discussion Preparation of ITZ-NLCs}

In this study, ITZ-NLCs were prepared using a hot homogenization method. This method involves the mixing of hot oil-in-water emulsion using a stirrer, followed by passage through a HPH device (EmulsiFlex-C3) to break down the particle size. ${ }^{4,26}$ The pressure for the HPH was set at 1,500 bar, and the sample was passed through for five cycles. Further increase in the number of cycles did not show any significant reduction in the particle size. The pressure and number of cycles were optimized to ensure the ITZ-NLCs achieved submicron size.

Surfactants are important for the stabilization of emulsions. TW80 has a hydrophilic-lipophilic balance value of 15 , whereas for Solutol HS15 it is between 14 and 16. ${ }^{2,27}$ TRA is a solvent for poorly water soluble drugs. It has a hydrophilic-lipophilic balance value of 4.2 and is commonly used for its surfactant properties in formulations. ${ }^{28}$ Therefore, TRA was added to our formulations to increase the solubility of ITZ in the lipid matrix.

\section{Physiochemical properties of lipid nanoparticles}

Preliminary studies were carried out to determine the amount of ITZ entrapped in the NLCs by breaking them down by heating and then extracting the drug. The method was similar to that reported by Hu et al..$^{29}$ The ITZ-NLCs were dissolved in ethanol heated to a temperature of $65^{\circ} \mathrm{C}$ in a water bath for 20 minutes and cooled to room temperature to precipitate 
the lipid. The sample was then centrifuged at 5,000 rpm for 15 minutes; the supernatant was collected; and the ITZ concentration was measured using the validated HPLC method. The results obtained with this method were similar to those obtained with the method described previously, in which the free drug concentration was measured first and then subtracted from the total drug concentration to determine the amount of entrapped drug. The subtraction method was used to calculate the amount of entrapped drug.

Several factors, including surfactant type and concentration, were varied to obtain the optimal ITZ EE. Surfactant plays an important role for NLC preparation, because it will influence the particle size, stability, crystallization behavior, and release profile. ${ }^{30}$ The physiochemical properties of ITZ-NLCs included EE, particle size, polydispersity index, and ZP were investigated for our formulations; the results are listed in Table 2.

Polydispersity index measures the size distribution of the nanoparticles in a sample. Monodispersed samples have a size distribution ranging from 0.01 to 0.7 , while samples with a broad distribution have a value of more than $0.7 .{ }^{31}$ All the batches made in this study had a polydispersity index value between 0.5 to 0.7 , suggesting that they were monodispersed. The measurement of the ZP represents the stability of the colloidal dispersion. Particles of the same charge with high ZP create repulsion to prevent flocculation, thereby maintaining the stability of the product. It has been suggested that a minimum $\mathrm{ZP}$ of less than $-60 \mathrm{mV}$ is required for excellent stability, and less than $-30 \mathrm{mV}$ for good physical stability. ${ }^{32}$ The formulations produced in this study were found to have a negative $\mathrm{ZP}$ between $-18.7 \mathrm{mV}$ and $-25.3 \mathrm{mV}$.

Table 2 Physical characteristics of formulation batches $(n=3)$

\begin{tabular}{lllll}
\hline Formulation & $\begin{array}{l}\text { Average } \\
\text { particle } \\
\text { size }(\mathbf{n m})\end{array}$ & PDI & ZP (mV) & EE (\%) \\
\hline FI & $354.5 \pm 14.0$ & $0.455 \pm 0.053$ & $-22.7 \pm 1.0$ & $59.5 \pm 2.3$ \\
F2 & $466.9 \pm 7.2$ & $0.568 \pm 0.055$ & $-25.3 \pm 0.7$ & $67.3 \pm 3.1$ \\
F3 & $414.1 \pm 39.2$ & $0.584 \pm 0.064$ & $-24.2 \pm 0.3$ & $65.4 \pm 2.5$ \\
F4 & $267.6 \pm 5.7$ & $0.467 \pm 0.025$ & $-19.6 \pm 0.8$ & $57.1 \pm 1.8$ \\
F5 & $404.8 \pm 21.4$ & $0.528 \pm 0.028$ & $-21.7 \pm 1.1$ & $70.8 \pm 2.0$ \\
F6 & $351.3 \pm 4.8$ & $0.355 \pm 0.035$ & $-18.9 \pm 0.4$ & $64.2 \pm 2.6$ \\
F7 & $469.6 \pm 18.5$ & $0.429 \pm 0.013$ & $-20.6 \pm 0.2$ & $63.1 \pm 1.9$ \\
F8 & $396.9 \pm 9.8$ & $0.445 \pm 0.05 I$ & $-23.8 \pm 0.4$ & $69.0 \pm 2.1$ \\
F9 & $340.6 \pm 6.7$ & $0.457 \pm 0.065$ & $-22.6 \pm 0.8$ & $66.3 \pm 0.4$ \\
FI0 & $310.5 \pm 15.2$ & $0.499 \pm 0.089$ & $-19.3 \pm 0.8$ & $64.7 \pm 2.3$ \\
FII & $358.9 \pm 23.6$ & $0.636 \pm 0.008$ & $-18.8 \pm 1.0$ & $72.6 \pm 1.3$ \\
FI2 & $313.7 \pm 15.3$ & $0.562 \pm 0.070$ & $-18.7 \pm 0.3$ & $70.5 \pm 0.6$ \\
\hline Abbris &
\end{tabular}

Abbreviations: PDI, polydispersity index; ZP, zeta potential; EE, entrapment efficiency.
When the concentration of TW80, HS15, or both was increased from $2 \%$ to $4 \%$, there was a reduction in the particle size. A similar finding was reported by Liu et al during the preparation of isotretinoin-loaded SLNs; their results suggested that, when the concentration of TW80 increased, the particle size became smaller. ${ }^{33}$ This is to be expected, because a higher surfactant concentration will cover the surface of the lipid phase, resulting in a smaller particle size. ${ }^{34}$

Reports in the literature have shown that increasing the surfactant concentration increased the EE for lipid nanoparticles. This was demonstrated in the study by Das et al, who found that when the surfactant concentration was increased from $0.5 \% \mathrm{w} / \mathrm{w}$ to $3 \% \mathrm{w} / \mathrm{w}$, there was an increase in tretinoin $\mathrm{EE}$ from $64.08 \% \pm 4.22 \%$ to $80.79 \% \pm 4.35 \%$. The presence of a sufficient amount of surfactant was proposed to help the solubilization and stabilization of drug molecules within the lipid matrix and at the surface. ${ }^{35}$ Similar findings were reported by Liu et al, who observed that increasing soybean lecithin concentration from $4 \% \mathrm{w} / \mathrm{w}$ to $8 \% \mathrm{w} / \mathrm{w}$ resulted in an increase in isotretinoin EE from $82.62 \% \pm 0.42 \%$ to $99.7 \% \pm 0.27 \% .{ }^{33}$ However, in this present study, the influence of surfactant concentration on EE was not significant.

\section{Transmission electron microscopy}

The transmission electron microscope image in Figure 1 shows that the ITZ-NLCs were spherical in shape, with a size between $150 \mathrm{~nm}$ and $200 \mathrm{~nm}$. Measurement using photon correlation spectroscopy does not measure the actual size, but only estimates the size of the nanoparticles based on

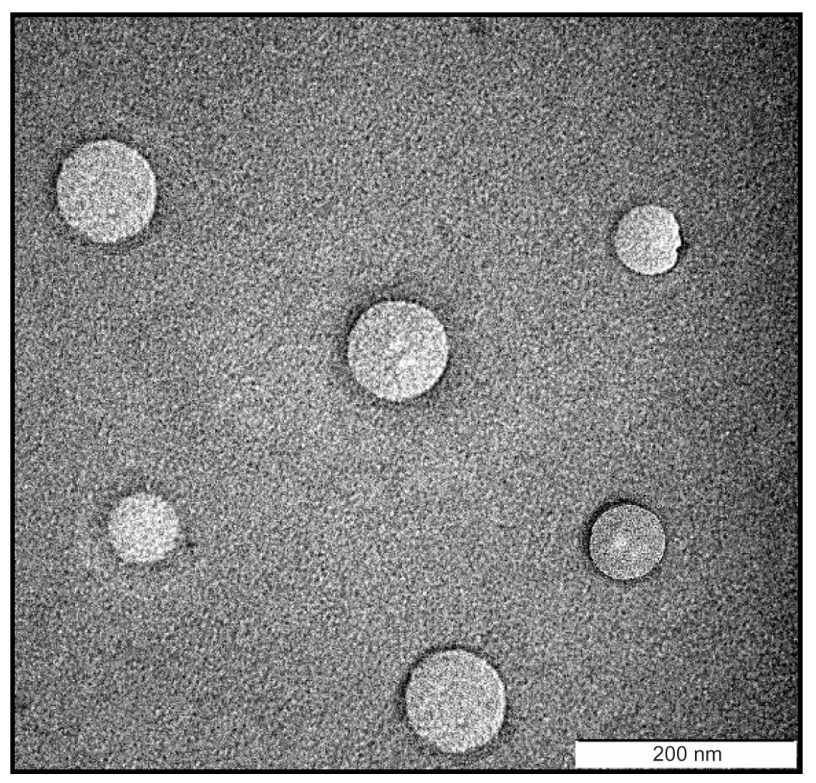

Figure I Transmission electron microscope image of itraconazole-loaded nanostructured lipid carriers. 
scattered light and intensity; ${ }^{3}$ therefore, transmission electron microscope analysis is useful for confirming the results from photon correlation spectroscopy and also in investigating the morphology of ITZ-NLCs.

\section{DSC}

DSC is a useful tool for studying the degree of crystallinity of a lipid. It is important to understand the crystallinity of a dosage form because it influences the drug-loading and drug-release patterns. ${ }^{4}$ During the formulation, the melting and cooling process can affect the polymorphic state of the lipid, changing from an unstable $\alpha$-state, to a metastable $\beta$ '-state, to stable $\beta$-states. ${ }^{36}$

The results for the DSC thermogram are shown in Figure 2. It was noted that the melting endothermic peaks for ITZ and Precirol ATO 5 were $167.77^{\circ} \mathrm{C}$ and $66.27^{\circ} \mathrm{C}$, respectively. However the melting peak for blank NLCs was reduced to $65.10^{\circ} \mathrm{C}$. This was due to their smaller particle size and higher surface area and the presence of surfactant. ${ }^{37,38}$ For ITZ-NLCs, the melting point was further reduced to $63.79^{\circ} \mathrm{C}$ due to the disordered crystal structure arrangement because of the incorporation of ITZ into the lipid matrix. The sharp endothermic peak for ITZ disappeared in ITZ-NLCs, and this suggests that ITZ was in an amorphous state. Similar findings were reported by Lv et al for DSC analysis of penciclovir-loaded SLNs. ${ }^{38}$ The endothermic peak for penciclovir was reported at $279^{\circ} \mathrm{C}$. However, when penciclovir-loaded SLNs were analyzed, the penciclovir peak was not found, and this showed that penciclovir existed in amorphous phase in their findings.

\section{XRD study}

The results for XRD analysis are shown in Figure 3. The sharp peaks for ITZ indicate the crystalline structure of ITZ. When blank NLCs were prepared without ITZ, the sharp peaks were not found because the pure lipid had lost its crystalline structure. For ITZ-NLCs, sharp peaks from ITZ were not present in the XRD pattern, which indicated that the ITZ was no longer in the crystalline form but in its amorphous form. ${ }^{7}$ Furthermore, the ITZ-NLCs had a lessordered crystal structure, which would contribute to a higher drug-loading capacity. ${ }^{39}$

A similar finding was reported by Chen et al for the XRD analysis of ITZ-loaded nanoparticles with human serum albumin. ${ }^{7}$ The XRD of ITZ powder also showed sharp peaks, which demonstrated the existence of crystalline ITZ. However, the XRD of ITZ nanoparticles only showed broad humps of amorphous material with no noticeable sharp peak of ITZ. This indicates that the ITZ was in the amorphous state in the human serum albumin nanoparticles.

\section{In vitro drug release study}

Formulation batch F12 was selected for study of the in vitro release profile; the methodology for this study was adapted from Patel et al. ${ }^{40}$

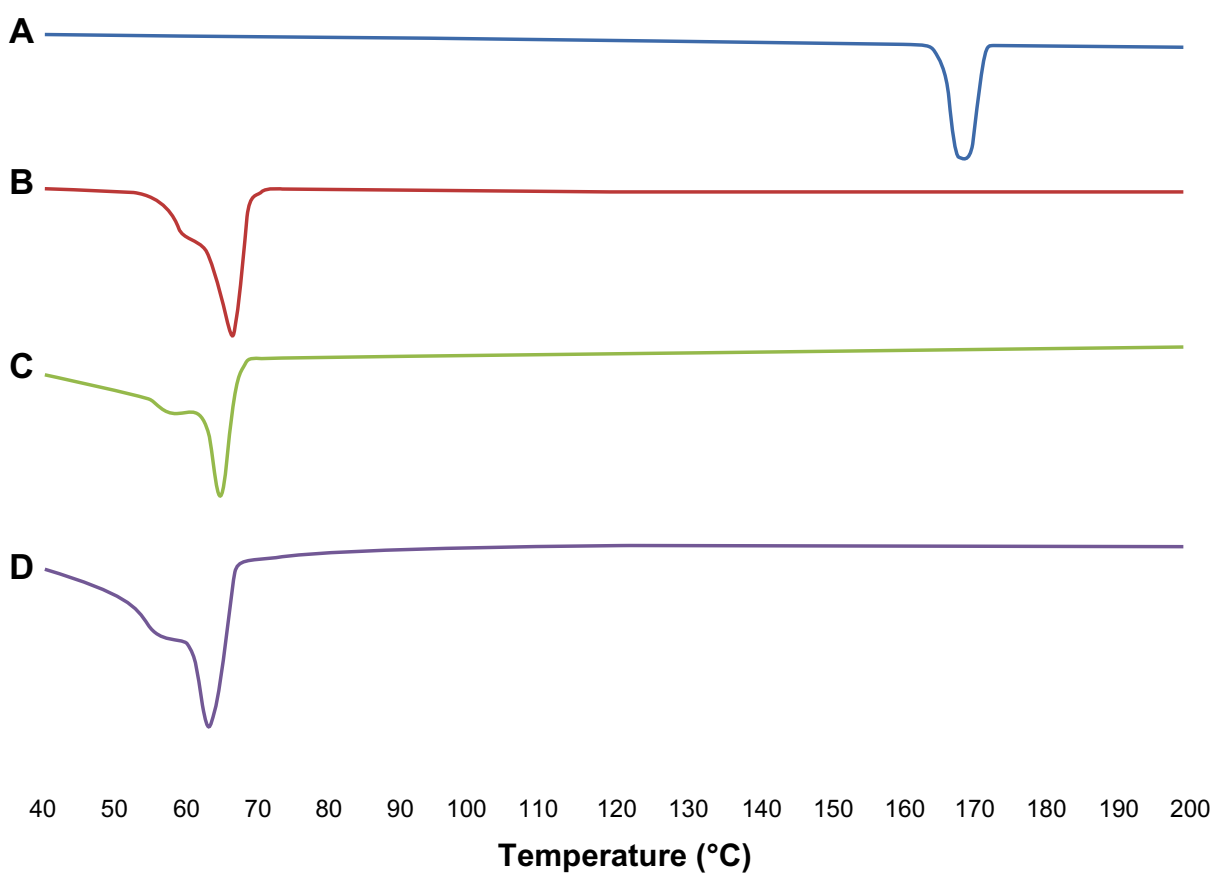

Figure 2 Differential scanning calorimetry analysis for ITZ, Precitol ATO 5, blank NLCs and ITZ-NLCs.

Notes: (A) Itraconazole; (B) Precirol ${ }^{\circledR}$ ATO 5 (Gattefossé Lyon, France); (C) blank nanostructured lipid carriers; (D) itraconazole-loaded nanostructured lipid carriers. Abbreviations: ITZ, itraconazole; ITZ-NLCs, itraconazole loaded nanostructured lipid carriers; NLCs, nanostructured lipid carriers. 


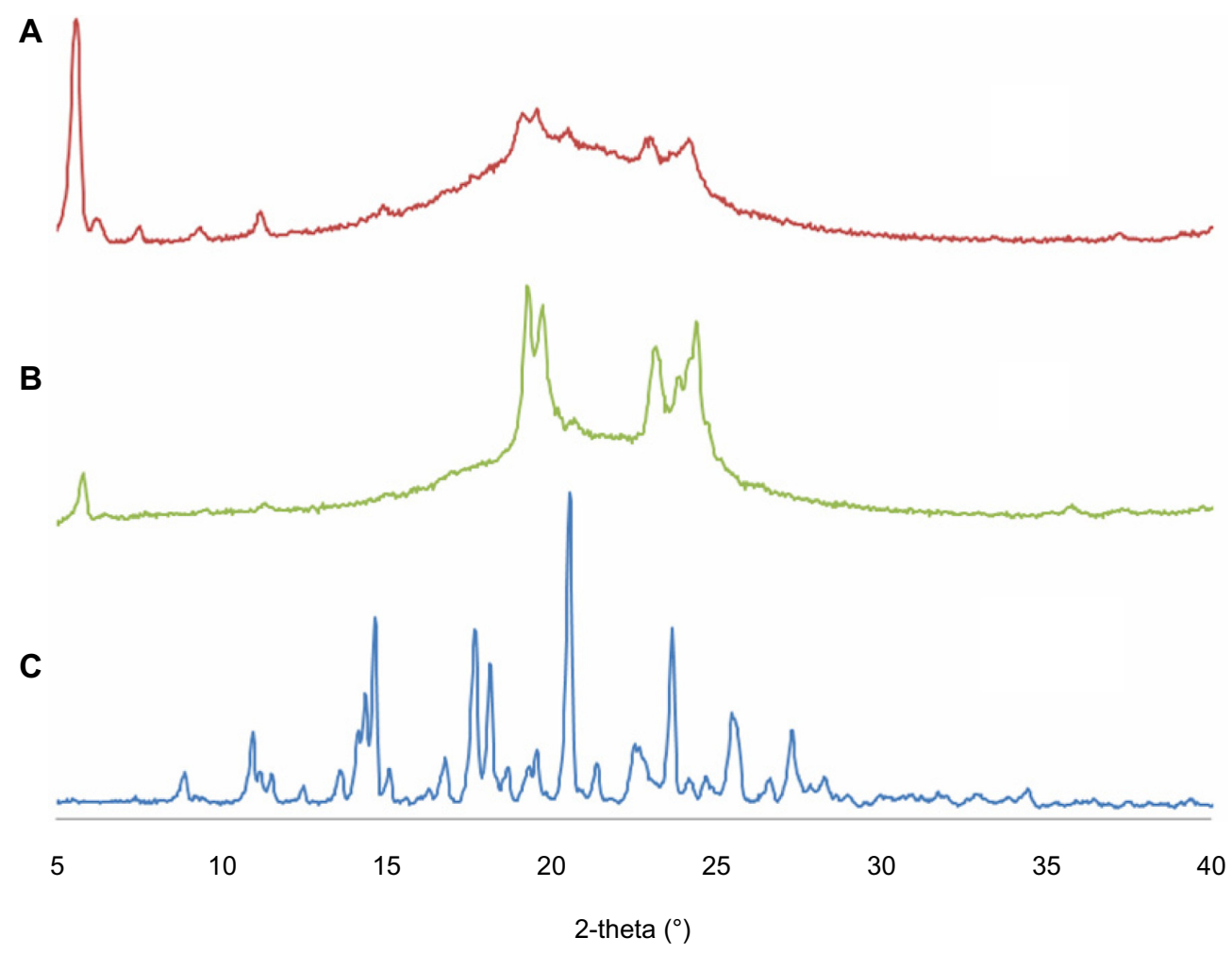

Figure 3 X-ray diffractogram for ITZ-NLCs, blank NLCs and ITZ.

Notes: (A) Itraconazole-loaded nanostructured lipid carriers; (B) blank nanostructured lipid carriers; (C) itraconazole.

Abbreviations: ITZ, itraconazole; ITZ-NLCs, itraconazole loaded nanostructured lipid carriers; NLCs, nanostructured lipid carriers.

The ITZ-NLCs exhibited a sustained-release profile up to 24 hours, with a total release of $80.6 \% \pm 5.3 \%$ as shown in Figure 4. A burst effect was observed with ITZ-NLCs, with $22.0 \% \pm 1.2 \%$ released in the first hour, followed by sustained-release to 24 hours. In contrast, the release for ITZ solution was much faster, achieving $95.0 \% \pm 2.53 \%$ within 20 minutes, indicating the permeability of ITZ through the dialysis membrane used.

The release pattern for NLCs depends on the nature of lipid matrix, surfactant concentration, and production temperature. This is best explained by the partitioning effects of the drug in both aqueous and lipid phases. During the formulation, increased temperature and the presence of surfactant will increase the drug solubility in the aqueous phase. During the cooling process, the drug will repartition into the lipid phase. The solid lipid recrystallizes, forming a solid lipid core, and the drug will deposit at the shell and/or on the surface of the lipid nanoparticles. Therefore, the presence of drug on the surface and outer shell contributes to the burst release, followed by sustained release by the drug incorporated in the lipid matrix. ${ }^{3}$

$\mathrm{Xie}$ et al reported that the in vitro release profile of enrofloxacin-loaded SLNs showed a biphasic drug-release pattern, with a burst release in the initial 2 hours, followed by a sustained release. ${ }^{41}$ In another study, Priyanka and Sathali formulated montelukast-loaded SLNs using three different lipids. All three lipids exhibited a burst release within 30 minutes, followed by sustained release. ${ }^{42}$

Based on the in vitro release results, we hypothesized that ITZ-NLCs were more likely to be the shell-enriched model, because most of the drug molecules were encapsulated at the lipid outer layer, which resulted in the immediate release followed by a slower release from the drug molecules in the lipid core.

\section{In vivo brain ITZ concentration}

Formulation batch F12 was selected for in vivo study. The in vivo results showed that ITZ-NLCs achieved a higher brain concentration compared to ITZ with H $\beta C D$. Statistical analysis showed a significant difference between the two groups $(P<0.05)$. ITZ-NLCs achieved a peak concentration at 8 hours ( $506.55 \mathrm{ng} / 0.2 \mathrm{~g}$ of brain homogenate), which is almost twice that of the H $\beta C D$ formulation $(253.29 \mathrm{ng} / 0.2 \mathrm{~g}$ of brain homogenate), as shown in Figure 5.

Although the burst effect of ITZ-NLCs was noted in the in vitro studies after 1 hour, it did not correspond to a higher brain concentration after 2 hours. A possible explanation for this observation is that the free drug or the drug in the 


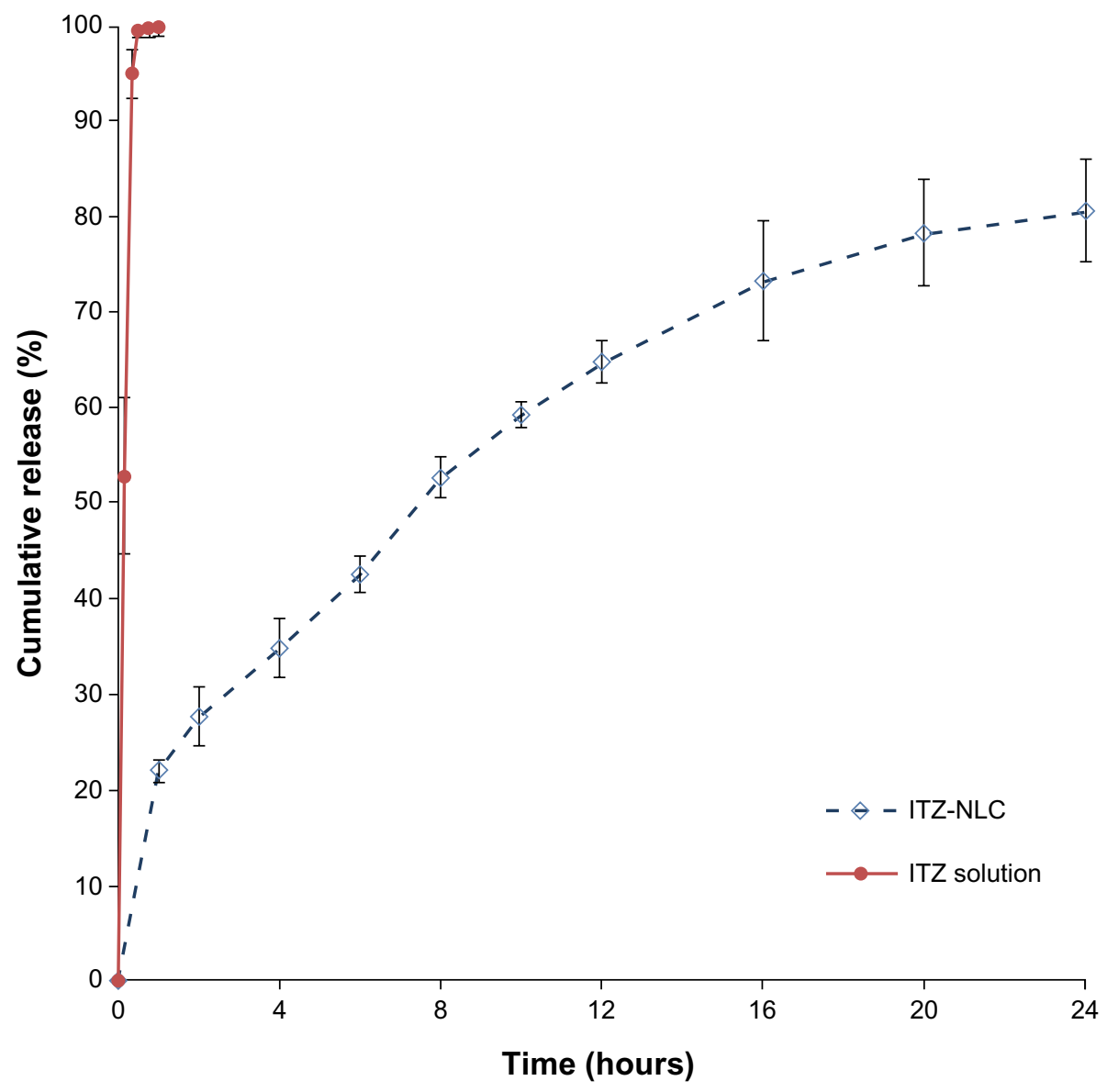

Figure 4 In vitro release profile for ITZ solution and ITZ-NLC formulation batch FI2 ( $=3$ ). Abbreviations: ITZ, itraconazole; ITZ-NLC, itraconazole-loaded nanostructured lipid carrier.

outer layer of the NLCs may have diffused into the blood circulation as free drug and thus could not permeate across the BBB.

It was noted that the brain concentration of ITZ gradually increased between 2 and 8 hours, with the highest concentration achieved at 8 hours. This may have been due to the permeation of ITZ-NLCs across the BBB as the drugs were released. The ITZ-NLCs may have permeated through the BBB and released the drug slowly over a period of time, causing the accumulation. A review paper reported that the presence of TW80 in the formulation of nanoparticles improved the permeability of nanoparticles across the BBB. ${ }^{17}$ It was reported that apolipoprotein $\mathrm{E}$ becomes adsorbed on the surface of nanoparticles containing TW80 and the complex binds to low-density lipoprotein receptor present on the BBB surface. ${ }^{17}$ In this case, the drugs are released upon endocytosis and transcytosis through the BBB endothelial cells.

Other studies have also reported the potential of lipid nanoparticles for brain delivery. Madan et al prepared noscapine-loaded SLNs conjugated with polyethylene glycol.
The results demonstrated that noscapine lipid nanoparticles not only increased the plasma half-life of noscapine, but were also able to cross the BBB and inhibit the proliferation of glioblastoma cells. ${ }^{43}$

In our study, the addition of TW80 to the formulation may have contributed to the permeability of ITZ across the BBB. The in vitro P-gp study using Caco- 2 cells, conducted by Zhang et al, demonstrated that the addition of TW80 to Caco- 2 cells was able to improve the permeability of digoxin, which is a substrate of P-gp, by inhibiting the efflux pump mechanism. ${ }^{44}$ Similar results were obtained in an in vivo study conducted in a xenograft mouse model of glioma by Estella-Hermoso de Mendoza et al using the anticancer drug edelfosine loaded into lipid nanoparticles. ${ }^{45}$ The results showed that the high level of edelfosine in the brain obtained with lipid nanoparticles correlated well with tumor growth suppression. This high edelfosine level was attributed to the inhibition of P-gp by TW80. ${ }^{45}$ Since ITZ is also a substrate of P-gp, the inhibition of P-gp by TW80 could be the reason for the improved delivery of ITZ across the BBB in the present study. 


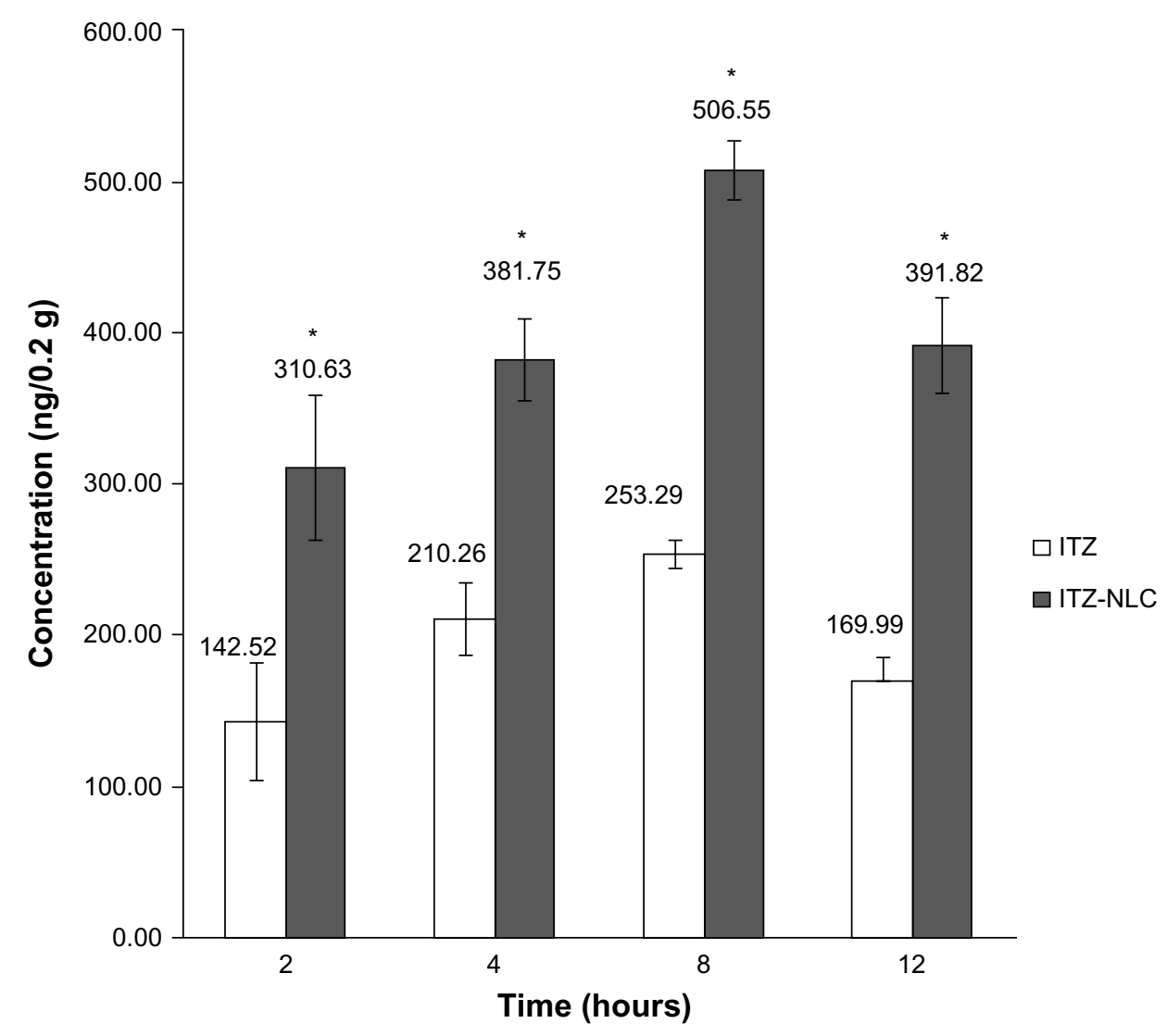

Figure 5 In vivo itraconazole concentration in brain $(n=3)$.

Note: $* p<0.05$.

Abbreviations: ITZ, itraconazole; ITZ-NLC, itraconazole-loaded nanostructured lipid carrier.

The small size of the lipid nanoparticles was able to prolong the circulation time in the body. Such prolongation increases the lipid nanoparticles' contact time with the $\mathrm{BBB}$ and also generates a concentration gradient across the BBB. ${ }^{18}$ Combining all the advantages offered by the lipid nanoparticles, the synergism might have improved the effective delivery of ITZ across the BBB.

\section{Conclusion}

In this study, ITZ-NLCs were successfully formulated and the physical characteristics analyzed. The in vivo study using mice has shown that ITZ-NLCs can improve the brain concentrations compared to the pure ITZ alone. Using lipid nanoparticles as a carrier and the addition of TW80 as surfactant may have contributed to the permeability of ITZ across the BBB. The results from this project show that NLC is a potential carrier for delivery of ITZ across the BBB.

\section{Acknowledgments}

The authors would like to thank Professor Peter Pook Chuen Keat from the International Medical University for his support, advice, and guidance for this project. We would also like to thank Professor Michael John Rathbone for the English correction and editing of this article.

\section{Disclosure}

The authors report no conflicts of interest in this work.

\section{References}

1. Wissing SA, Kayser O, Müller RH. Solid lipid nanoparticles for parenteral drug delivery. Adv Drug Deliv Rev. 2004;56(9):1257-1272.

2. Severino P, Andreani T, Macedo AS, et al. Current state-of-art and new trends on lipid nanoparticles (SLN and NLC) for oral drug delivery. J Drug Deliv. 2012;2012:750891

3. Müller RH, Mäder K, Gohla S. Solid lipid nanoparticles (SLN) for controlled drug delivery - a review of the state of the art. Eur J Pharm Biopharm. 2000;50(1):161-177.

4. Mehnert W, Mäder K. Solid lipid nanoparticles: production, characterization and applications. Adv Drug Deliv Rev. 2001;47(2-3):165-196.

5. Müller RH, Radtke M, Wissing SA. Solid lipid nanoparticles (SLN) and nanostructured lipid carriers (NLC) in cosmetic and dermatological preparations. Adv Drug Deliv Rev. 2002;54 Suppl 1:S131-S155.

6. Müller RH, Radtke M, Wissing SA. Nanostructured lipid matrices for improved microencapsulation of drugs. Int J Pharm. 2002;242(1-2): 121-128.

7. Chen W, Gu B, Wang H, Pan J, Lu W, Hou H. Development and evaluation of novel itraconazole-loaded intravenous nanoparticles. Int J Pharm. 2008;362(1-2):133-140. 
8. Maertens J, Boogaerts M. The place for itraconazole in treatment. J Antimicrob Chemother. 2005;56 Suppl 1:i33-i38.

9. De Beule K. Itraconazole: pharmacology, clinical experience and future development. Int J Antimicrob Agents. 1996;6(3):175-181.

10. Pardeike J, Weber S, Haber T, et al. Development of an itraconazoleloaded nanostructured lipid carrier (NLC) formulation for pulmonary application. Int J Pharm. 2011;419(1-2):329-338.

11. Peeters J, Neeskens P, Tollenaere JP, Van Remoortere P, Brewster ME. Characterization of the interaction of 2-hydroxypropyl-betacyclodextrin with itraconazole at $\mathrm{pH} \mathrm{2,} \mathrm{4,} \mathrm{and} \mathrm{7.} \mathrm{J} \mathrm{Pharm} \mathrm{Sci.} \mathrm{2002;}$ 91(6): 1414-1422.

12. Akkar A, Müller RH. Intravenous itraconazole emulsions produced by SolEmuls technology. Eur J Pharm Biopharm. 2003;56(1):29-36.

13. Kim JK, Park JS, Kim CK. Development of a binary lipid nanoparticles formulation of itraconazole for parenteral administration and controlled release. Int J Pharm. 2010;383(1-2):209-215.

14. Kethireddy S, Andes D. CNS pharmacokinetics of antifungal agents. Expert Opin Drug Metab Toxicol. 2007;3(4):573-581.

15. Pardridge WM. Blood-brain barrier delivery. Drug Discov Today. 2007;12(1-2):54-61.

16. Hawkins BT, Davis TP. The blood-brain barrier/neurovascular unit in health and disease. Pharmacol Rev. 2005;57(2):173-185.

17. Blasi P, Giovagnoli S, Schoubben A, Ricci M, Rossi C. Solid lipid nanoparticles for targeted brain drug delivery. Adv Drug Deliv Rev. 2007;59(6):454-477.

18. Kaur IP, Bhandari R, Bhandari S, Kakkar V. Potential of solid lipid nanoparticles in brain targeting. $J$ Control Release. 2008;127(2): 97-109.

19. Gabathuler R. Approaches to transport therapeutic drugs across the blood-brain barrier to treat brain diseases. Neurobiol Dis. 2010;37(1): $48-57$.

20. Pardridge WM. Blood-brain barrier drug targeting: the future of brain drug development. Mol Interv. 2003;3(2):90-105, 51

21. Miyama T, Takanaga H, Matsuo H, et al. P-glycoprotein-mediated transport of itraconazole across the blood-brain barrier. Antimicrob Agents Chemother. 1998;42(7):1738-1744.

22. Duret C, Wauthoz N, Sebti T, Vanderbist F, Amighi K. New inhalationoptimized itraconazole nanoparticle-based dry powders for the treatment of invasive pulmonary aspergillosis. Int J Nanomedicine. 2012;7: 5475-5489.

23. Yang SC, Lu LF, Cai Y, Zhu JB, Liang BW, Yang CZ. Body distribution in mice of intravenously injected camptothecin solid lipid nanoparticles and targeting effect on brain. J Control Release. 1999;59(3): 299-307.

24. Zara GP, Cavalli R, Fundarò A, Bargoni A, Caputo O, Gasco MR. Pharmacokinetics of doxorubicin incorporated in solid lipid nanospheres (SLN). Pharmacol Res. 1999;40(3):281-286.

25. Redmann S, Charles BG. A rapid HPLC method with fluorometric detection for determination of plasma itraconazole and hydroxy-itraconazole concentrations in cystic fibrosis children with allergic bronchopulmonary aspergillosis. Biomed Chromatogr. 2006;20(4): 343-348.

26. Noack A, Hause G, Mader K. Physicochemical characterization of curcuminoid-loaded solid lipid nanoparticles. Int J Pharm. 2012;423(2): $440-451$.

27. Vandamme TF, Anton N. Low-energy nanoemulsification to design veterinary controlled drug delivery devices. Int $J$ Nanomedicine. 2010;5:867-873.

International Journal of Nanomedicine

\section{Publish your work in this journal}

The International Journal of Nanomedicine is an international, peerreviewed journal focusing on the application of nanotechnology in diagnostics, therapeutics, and drug delivery systems throughout the biomedical field. This journal is indexed on PubMed Central, MedLine, CAS, SciSearch ${ }^{\circledR}$, Current Contents ${ }^{\circledR} /$ Clinical Medicine,
28. Baboota S, Shakeel F, Ahuja A, Ali J, Shafiq S. Design, development and evaluation of novel nanoemulsion formulations for transdermal potential of celecoxib. Acta Pharm. 2007:57(3):315-332.

29. Hu FQ, Yuan H, Zhang HH, Fang M. Preparation of solid lipid nanoparticles with clobetasol propionate by a novel solvent diffusion method in aqueous system and physicochemical characterization. Int J Pharm. 2002;239(1-2):121-128.

30. Vitorino C, Carvalho FA, Almeida AJ, Sousa JJ, Pais AA. The size of solid lipid nanoparticles: an interpretation from experimental design. Colloids Surf B Biointerfaces. 2011;84(1):117-130.

31. Nidhin M, Indumathy R, Sreeram K, Nair B. Synthesis of iron oxide nanoparticles of narrow size distribution on polysaccharide templates. Bulletin of Materials Science. 2008;31:93-96.

32. Freitas C, Müller RH. Effect of light and temperature on zeta potential and physical stability in solid lipid nanoparticle (SLN $\left.{ }^{\mathrm{TM}}\right)$ dispersions. Int J Pharm. 1998;168(2):221-229.

33. Liu J, Hu W, Chen $\mathrm{H}, \mathrm{Ni} \mathrm{Q}, \mathrm{Xu} \mathrm{H}$, Yang X. Isotretinoin-loaded solid lipid nanoparticles with skin targeting for topical delivery. Int J Pharm 2007;328(2):191-195.

34. Helgason T, Awad TS, Kristbergsson K, McClements DJ, Weiss J. Effect of surfactant surface coverage on formation of solid lipid nanoparticles (SLN). J Colloid Interface Sci. 2009;334(1):75-81.

35. Das S, Ng WK, Kanaujia P, Kim S, Tan RB. Formulation design, preparation and physicochemical characterizations of solid lipid nanoparticles containing a hydrophobic drug: effects of process variables. Colloids Surf B Biointerfaces. 2011;88(1):483-489.

36. Silva AC, González-Mira E, García ML, et al. Preparation, characterization and biocompatibility studies on risperidone-loaded solid lipid nanoparticles (SLN): high pressure homogenization versus ultrasound. Colloids Surf B Biointerfaces. 2011;86(1):158-165.

37. Venkateswarlu V, Manjunath K. Preparation, characterization and in vitro release kinetics of clozapine solid lipid nanoparticles. J Control Release. 2004;95(3):627-638.

38. Lv Q, Yu A, XiY, et al. Development and evaluation of penciclovir-loaded solid lipid nanoparticles for topical delivery. Int J Pharm. 2009;372(1-2):191-198.

39. Abdelbary G, Fahmy RH. Diazepam-loaded solid lipid nanoparticles: design and characterization. AAPS PharmSciTech. 2009;10(1): 211-219.

40. Patel K, Padhye S, Nagarsenker M. Duloxetine HCl lipid nanoparticles: preparation, characterization, and dosage form design. AAPS PharmSciTech. 2012;13(1):125-133.

41. Xie S, Zhu L, Dong Z, et al. Preparation, characterization and pharmacokinetics of enrofloxacin-loaded solid lipid nanoparticles: influences of fatty acids. Colloids Surf B Biointerfaces. 2011;83(2):382-387.

42. Priyanka K, Sathali AA. Preparation and evaluation of montelukast sodium loaded solid lipid nanoparticles. J Young Pharm. 2012;4(3): 129-137.

43. Madan J, Pandey RS, Jain V, Katare OP, Chandra R, Katyal A. Poly (ethylene)-glycol conjugated solid lipid nanoparticles of noscapine improve biological half-life, brain delivery and efficacy in glioblastoma cells. Nanomedicine. 2013;9(4):492-503.

44. Zhang H, Yao M, Morrison RA, Chong S. Commonly used surfactant, Tween 80, improves absorption of P-glycoprotein substrate, digoxin, in rats. Arch Pharm Res. 2003;26(9):768-772.

45. Estella-Hermoso de Mendoza A, Préat V, Mollinedo F, Blanco-Prieto MJ. In vitro and in vivo efficacy of edelfosine-loaded lipid nanoparticles against glioma. J Control Release. 2011;156(3):421-426.

\section{Dovepress}

Journal Citation Reports/Science Edition, EMBase, Scopus and the Elsevier Bibliographic databases. The manuscript management system is completely online and includes a very quick and fair peer-review system, which is all easy to use. Visit http://www.dovepress.com/ testimonials.php to read real quotes from published authors. 